\title{
Construction and Quality Control of Obstetrics and Gynecology Disease Biobank
}

Yanhong Liu, Hong Gao, Yue Hu, Meiling Ge, Jie Ding, Qing Ye* Corresponding author E-mail: nanjingbiobank@163.com

Biobank of Nanjing Drum Tower Hospital, The Affiliated Hospital of Nanjing University Medical School

Nanjing multi-center biobank , Jiangsu Biobank of Clinical Resources

Aim: To ensure the sample quality meet the requirement of experiment research, the gynecology and obstetrics biobank of Nanjing Drum Tower hospital designed different quality control method for relevant types of samples and a range of viable quality control procedure has been formulated.

Methods: The gynecology and obstetrics department and biobank cooperate to accomplish the biobanking process. Disease categories included birth defect, preeclampsia (PE), endometriosisand gynecological oncology. Sample types after disposition were frozen tissue, paraffinembedded tissue, OCT-embedded tissue, plasma, buffy coat, serum, blood clots and urine. Different categories of samples were randomly selected $1 \%$ cases for quality control experiments:

$\checkmark$ frozen tissue, buffy coat and blood clots: RNA and DNA were extracted and the concentration, purity and integrity were detected.

- paraffin-embedded tissue:

morphological observation after hematoxylin-eosinstaining and immunohistochemistry (IHC) detection of $\beta$-actin.

- OCT-embedded tissue:

immunofluorescence detection of $\beta$-actin.

- Four kind of organs (skin, heart, liverand placenta) frozen tissue samples derived from 18 fetal autopsy specimens were chosen for study of RNA quality between cold ischemia time(CIT).
Results: Recently there are about 2,000 cases, 360,000 vials samples in the obstetrics and gynecology disease biobank. According to the results of quality control experiments: 1) All the concentration and purity parameters of selected samples were qualified. 2) Frozen tissues with cold ischemia time $(\mathrm{CIT}) \leq 1 \mathrm{~h}$, using qualified standard $\mathrm{RIN} \geq 7$, the qualification rate was $90 \%$.

Tab1. Quality control experiment projects of different sample types.

\begin{tabular}{|c|c|c|}
\hline Sample types & experimentethods & detection index \\
\hline frozen tissue & $\begin{array}{l}\text { RNA extraction } \\
\text { RNA concentration and purity } \\
\text { detection } \\
\text { RNA integrity detection } \\
\text { RT-qPCR }\end{array}$ & $\begin{array}{l}\text { RNA concentration, } \\
\text { RNA purity, } \\
\text { RNA integrity } \\
\beta \text {-actin mRNA } \\
\text { expression } \\
\end{array}$ \\
\hline $\begin{array}{l}\text { buffy coat and } \\
\text { blood clots }\end{array}$ & $\begin{array}{l}\text { DNAextraction } \\
\text { DNA concentration and purity } \\
\text { detection } \\
\text { DNA integrity detection } \\
\end{array}$ & $\begin{array}{l}\text { DNA concentration, } \\
\text { DNA purity, } \\
\text { DNA integrity }\end{array}$ \\
\hline $\begin{array}{l}\text { paraffin-embedded } \\
\text { tissue }\end{array}$ & $\begin{array}{l}\text { morphological observation } \\
\text { hematoxylin-esin staining } \\
\text { immunohistochemistry }\end{array}$ & $\begin{array}{l}\text { Morphological images } \\
\beta \text {-tubulin protein } \\
\text { expression }\end{array}$ \\
\hline $\begin{array}{l}\text { OCT-embedded } \\
\text { tissue }\end{array}$ & immunofluorescence & $\begin{array}{l}\beta \text {-tubulin protein } \\
\text { expression }\end{array}$ \\
\hline
\end{tabular}

3) Frozen tissues with CIT between 1 18h, using qualified standard $R I N \geq 5$, the qualification rate was $61.1 \%$. 4) All the paraffin-embedded tissues were qualified for morphological observation 5) The qualification rate of OCT-embedded tissue was $89 \% .6$ ) CIT had a great influence on the integrity of frozen tissue RNA, and the RNA integrity parameters of different tissue types in the same specimen were significantly different.
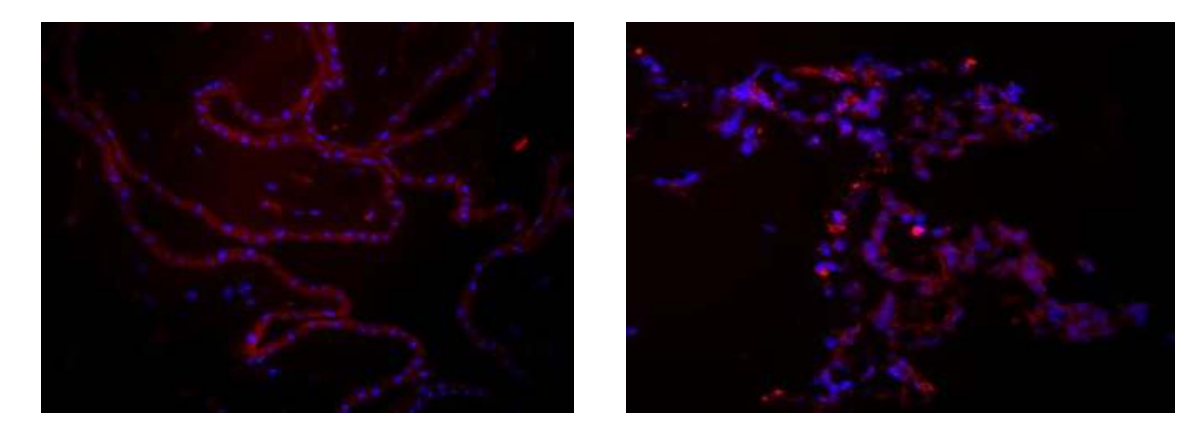

Fig.1 Images of mmunofluorescence detection of $\beta$ actin in endometrium tissue and placenta, 400x. Left: endometrium; Right: placenta
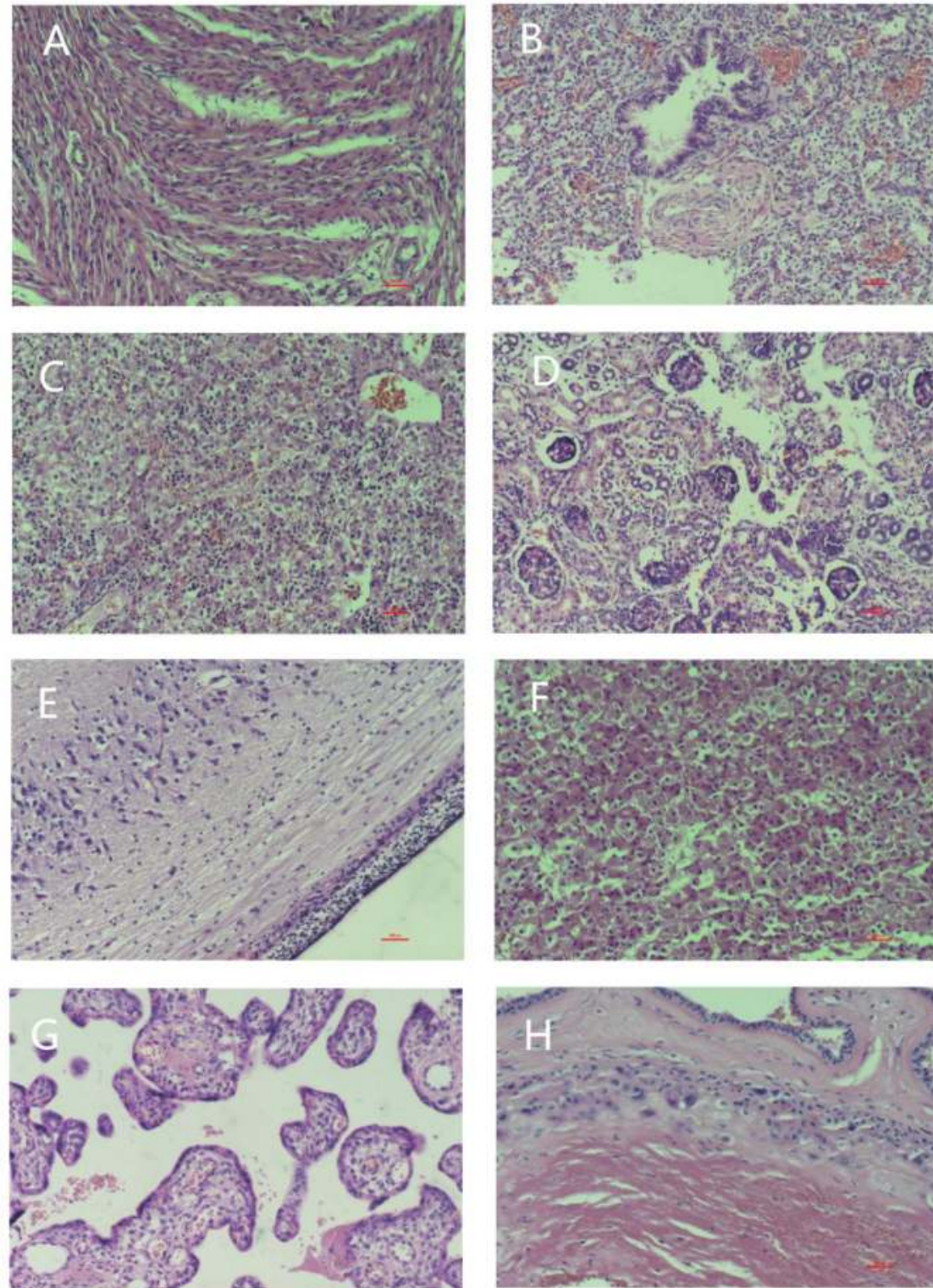

ig. 2 Hematoxylin-eosin staining images of multiple issues and different organs derived from autopsy. 200x. A) Heart B) Lung C)Liver D)Kidney E)Brain F)Adrenal gland G)Placenta $H$ ) Embryolemma
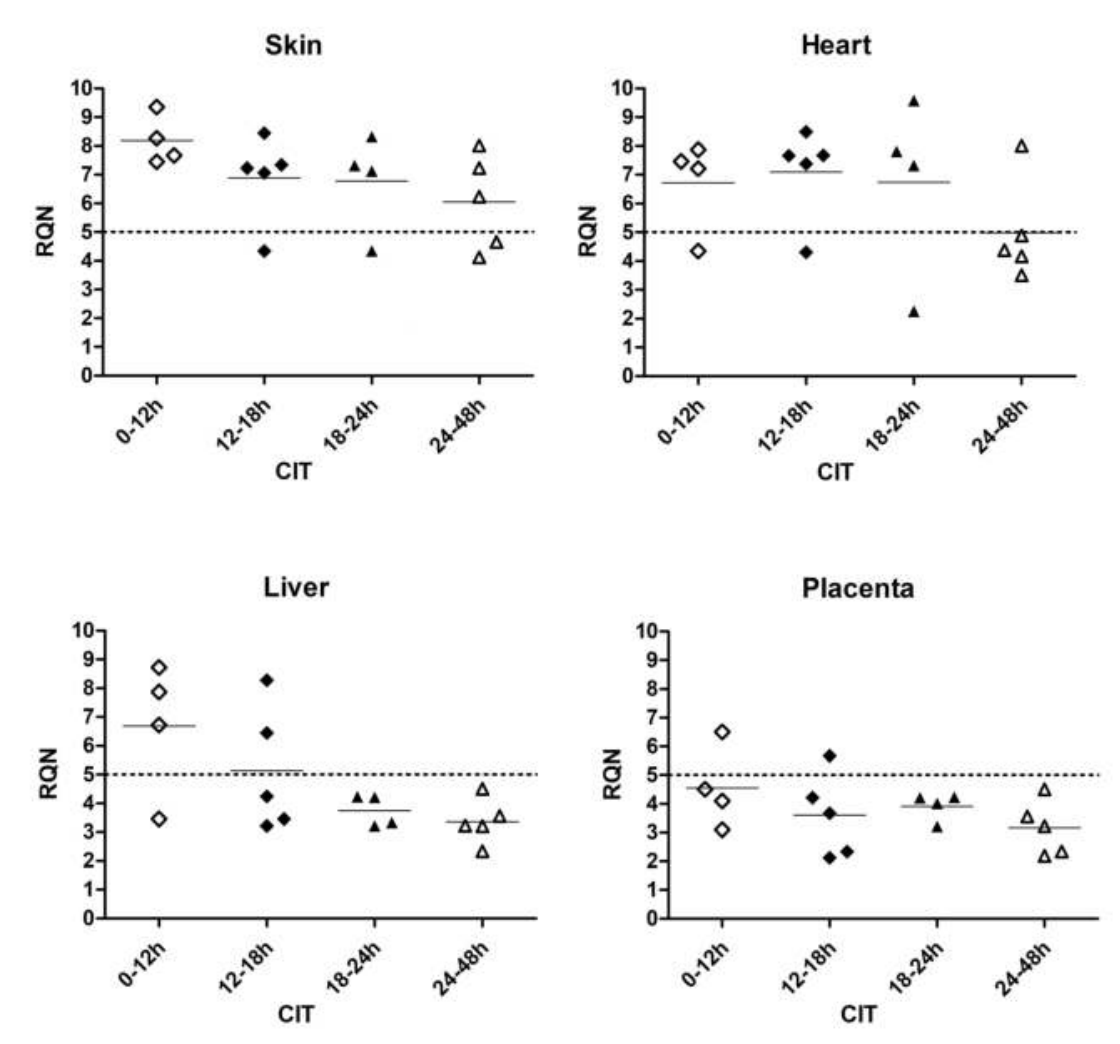

Conclusions: An obstetrics and gynecology disease biobank with various types of diseases and abundant samples was constructed. Using specific quality control experiments for different types of samples was a reliableoperating strategy, which can be beneficial for providing qualified research resources.

Key word: obstetrics and gynecology disease, biospecimen, biobanking, quality control, qualification rate

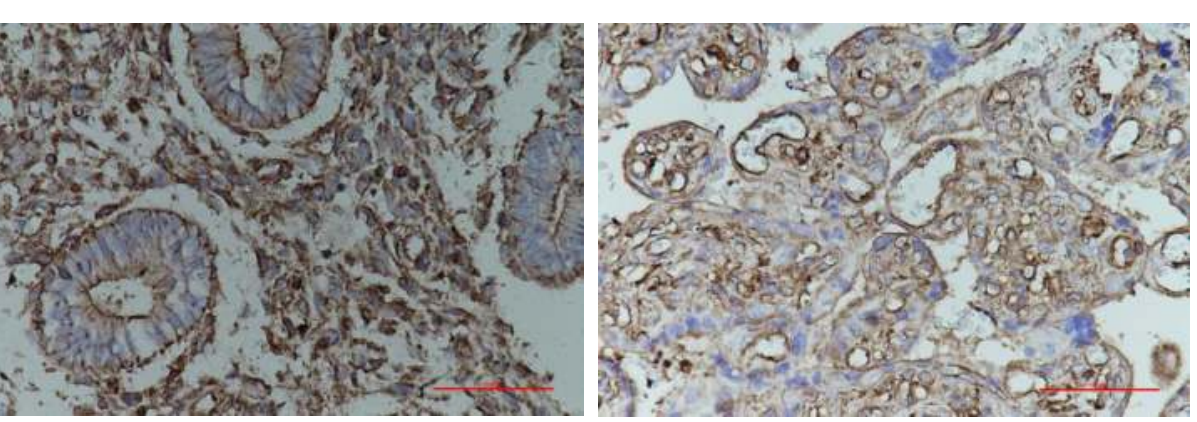

Fig. 3 Images of immunohistochemistry (IHC) detection of $\beta$-actin in endometrium tissue and placenta, 400x Left: endometrium; Right: placenta

Fig. 4 Left: The relationship between RQN and CIT in four different tissue types; Right: The relationship between RQN and tissue types in four groups of different CIT, the sort order from good to poor, which were skin, heart, liver and placenta.
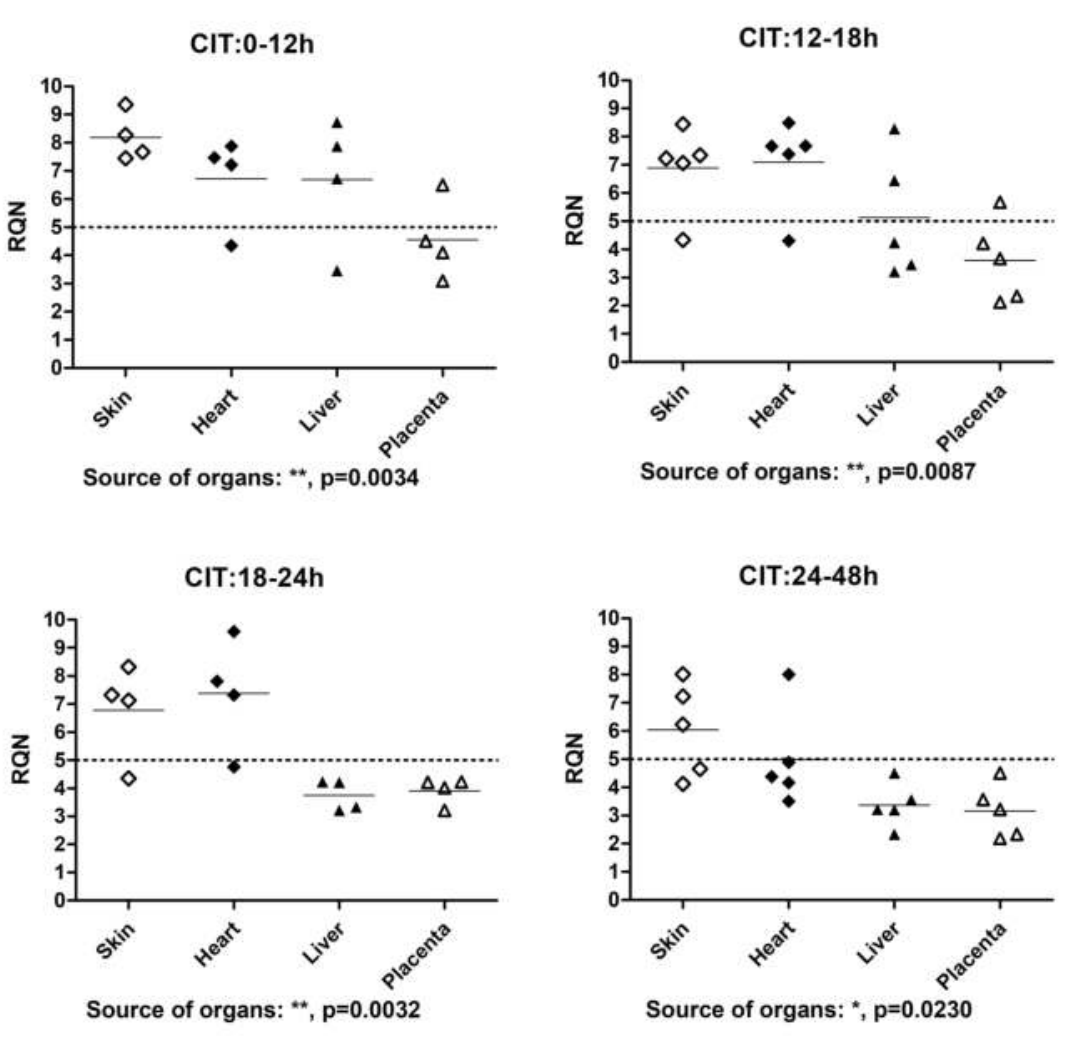